\title{
The implication of an advanced bioprocess for the acquisition of valuable microbial resources toward a sustainable and low-environmental burden society
}

\author{
Akihiko Terada ${ }^{1}$ \\ Published online: 16 June 2020 \\ ๑) Springer-Verlag GmbH Germany, part of Springer Nature 2020
}

One of the most exciting discoveries in the year 2019 in the field of environmental microbiology is the isolation and cultivation of Asgard archaeon present in deep-sea sediment (Imachi et al. 2020). The achievement has captured considerable attention of not only researchers but also the general public. According to the metagenomics study (Spang et al. 2015; Zaremba-Niedzwiedzka et al. 2017), Asgard archaea potentially possess genes that prokaryotes are not supposed to harbor. The metagenomic information has encouraged discussion whether the genes are derived from the Asgard archaeon per se, sample contamination, or metagenomic artifacts. The isolation and cultivation of an Asgard archaeon, termed 'Candidatus Prometheoarchaeum syntrophicum' strain MK-D1, seemingly brought the longterm discussion to an end-this Asgard archaeon does hold eukaryote-specific genes. The genome insights and physiological traits of the purely isolated archaeon allowed the authors to advocate the model to describe how eukaryotes emerged.

In addition to the groundbreaking achievement, readers with engineering backgrounds could be intrigued with potential methods to isolate an Asgard archaeon, which possesses an extremely slow-growing nature. The authors applied a fixed-film bioreactor technology with hanged polyurethane foam cubes in series and inoculated anaerobic deep-sea sediment to the bioreactor, allowing slow-growing microorganisms to be retained in the bioreactor. The continuous and intermittent supplies of liquid medium and $\mathrm{CH}_{4} /$ $\mathrm{CO}_{2}$ gas, respectively, for over 2000 days allowed the creation of a favorable environment for the growth of the strain MK-D1. The authors inferred that the successful enrichment

Akihiko Terada

akte@cc.tuat.ac.jp

1 Tokyo University of Agriculture and Technology, 2-24-16, Naka, Koganei, Tokyo 184-8588, Japan plausibly increased the isolation probability, shedding light on the significance of an enrichment procedure.

Such laborious but unsurpassed cultivation work reminds me of the tenet by a Dutch microbiologist, Lourens Gerhard Marinus Baas Becking (1895-1963), 'Everything is everywhere, but the environment selects.' Due to myriad discoveries of unexplored microorganisms in geographically and geologically distinct environments, we recognize that the former part may not explain reality as discussed elsewhere (De Wit and Bouvier 2006). Nevertheless, the latter part, 'but the environment selects', supports groundbreaking discoveries if an appropriate inoculum and favorable conditions for targeted microorganisms are met. Environmental and chemical engineering, the expertise of core readers in this journal, could contribute to this point: Environmental and chemical engineering could gear up for exploring beneficial microbes that are present but hidden in nature.

Indeed, the concept based on the enrichment of microorganisms with novel functions-regardless of the presence or absence of the ensuing isolation-has been implemented in the field of environmental engineering for a sustainable and low-environmental burden society. By developing and operating unique devices or well-controlled bioreactors, suitable conditions were provided to enrich, isolate, and culture targeted microorganisms at a laboratory level. For instance, a compartmentalized well device where a single bacterial cell can be grown in an indigenous environment allowed the cultivation of bacterium, excreting a highly active antibiotic compound, teixobactin (Ling et al. 2015). Bioreactors with precisely controlled environments enhanced the enrichment of novel bacteria with unique functions; e.g., fixing inert nitrogen, which could be an alternative way to ultimately replace the Harbor-Bosch process (Ospina-Betancourth et al. 2020), efficienty converting nitrous oxide, a highly potent greenhouse gas, into harmless nitrogen (Ishii et al. 2011; Suenaga et al. 2019), and producing proteins from industrial wastewaters (Alloul et al. 2019). These achievements 
indicate the significance of creating environments in which targeted microorganisms favorably thrive. The latter part of the tenet by 'but the environment selects'. makes sense to me.

Given that these discoveries are a tip of the iceberg on this planet where hidden microbial resources are always held, an untold number of microorganisms have yet remained unexplored for a few billion years. Enriching, isolating, and cultivating these microbes are still time-consuming and labor-intensive in general. Nevertheless, understanding their physiologies and mimicking favorable niches by an advanced bioreactor technology speeds up the enrichment of novel microorganisms and improves the probability of isolation success for applying to industries. To this end, the basics of environmental and process engineering would be conducible.

In sum, environmental and bioprocess engineers are poised to broaden their roles in controlling microenvironments for hunting undiscovered and beneficial microorganisms toward useful applications. This extended mission could facilitate bridging discoveries to building new platforms for sustainable society with low-environmental burden.

\section{References}

Alloul A, Wuyts S, Lebeer S, Vlaeminck SE (2019) Volatile fatty acids impacting phototrophic growth kinetics of purple bacteria: paving the way for protein production on fermented wastewater. Water Res 152:138-147

De Wit R, Bouvier T (2006) 'Everything is everywhere, but, the environment selects'; what did Baas Becking and Beijerinck really say? Environ Microbiol 8(4):755-758

Imachi H, Nobu MK, Nakahara N, Morono Y, Ogawara M, Takaki Y et al (2020) Isolation of an archaeon at the prokaryote-eukaryote interface. Nature 577:519-525

Ishii S, Ohno H, Tsuboi M, Otsuka S, Senoo K (2011) Identification and isolation of active $\mathrm{N}_{2} \mathrm{O}$ reducers in rice paddy soil. ISME J 5(12):1936-1945

Ling LL, Schneider T, Peoples AJ, Spoering AL, Engels I, Conlon BP et al (2015) A new antibiotic kills pathogens without detectable resistance. Nature 517:455-459

Ospina-Betancourth C, Acharya K, Allen B, Entwistle J, Head IM, Sanabria J, Curtis TP (2020) Enrichment of nitrogen-fixing bacteria in a nitrogen-deficient wastewater treatment system. Environ Sci Technol 54:3539-3548

Spang A, Saw JH, Jørgensen SL, Zaremba-Niedzwiedzka K, Martijn J, Lind AE et al (2015) Complex archaea that bridge the gap between prokaryotes and eukaryotes. Nature 521:173-179

Suenaga T, Hori T, Riya S, Hosomi M, Smets BF, Terada A (2019) Enrichment, Isolation, and characterization of high-affinity $\mathrm{N}_{2} \mathrm{O}$-reducing bacteria in a gas-permeable membrane reactor. Environ Sci Technol 53:12101-12112

Zaremba-Niedzwiedzka K, Caceres EF, Saw JH, Bäckström D, Juzokaite L, Vancaester E et al (2017) Asgard archaea illuminate the origin of eukaryotic cellular complexity. Nature 541:353-358

Publisher's Note Springer Nature remains neutral with regard to jurisdictional claims in published maps and institutional affiliations. 